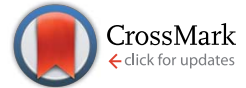

Cite this: RSC Adv., 2015, 5, 56162
Received 3rd April 2015 Accepted 3rd June 2015

DOI: $10.1039 / \mathrm{c} 5 \mathrm{ra05997f}$

www.rsc.org/advances

\section{IR 820 stabilized multifunctional polycaprolactone glycol chitosan composite nanoparticles for cancer therapy $\dagger$}

\begin{abstract}
Piyush Kumar and Rohit Srivastava*
Photothermal therapy has gained worldwide attention for its less painful, non invasive/minimally invasive, effective thermal ablation based therapy for cancer. It has been found to be effective in the treatment of drug-resistant cancer. Currently available photothermal systems have several limitations such as a high cost, incompatibility and clearance from the physiological system. To address these issues, we have prepared multifunctional biocompatible and biodegradable cost effective IR 820 dye encapsulated glycol chitosan coated polycaprolactone composite nanoparticles (PCLGC-IR) with improved performance. Polycaprolactone (PCL) has been used as a cheap biodegradable polymer, glycol chitosan (GC) as an immunoadjuvant for immune response generation followed by the cost-effective IR 820 dye for photothermal therapy. IR 820 encapsulation increases the stability of the PCLGC-IR nanoparticles as confirmed by DSC and XRD, while the glycol chitosan enhances the uptake of the composite nanoparticles by MDA-MB-231 breast cancer cells. The PCLGC-IR composite nanoparticles with a size of around 150-200 nm can be irradiated with a dual laser at $750 \mathrm{~nm}$ and $808 \mathrm{~nm}$ in photothermal therapy (PTT). In addition to PTT, the PCLGC-IR composite nanoparticles can also be used for sustainable controlled drug release as they retain their structural integrity even after laser treatment. Further we analyzed the degraded product using liquid chromatography mass spectroscopy (LCMS) upon photo irradiation. A cytotoxicity study of the PCLGC-IR composite nanoparticles was carried out on NIH3T3 cells. Fluorescence microscope image analysis of MDA-MB-231 cells showed hyperthermic cell death via apoptosis and necrosis due to an enhanced uptake of the PCLGC-IR composite nanoparticles. Our results showed that this nanoformulation can be used for the effective treatment of multiple drug resistant cancer.
\end{abstract}

\section{Introduction}

Photothermal therapy (PTT) has become popular in modern cancer therapy due to its non-invasive/minimally invasive features compared to surgery. PTT uses a near infra-red (NIR) absorbing material such as gold, carbon nanotubes, graphene oxides or dyes. ${ }^{1-5}$ These NIR materials, upon photo irradiation, generate heat. A rise in temperature to $>42{ }^{\circ} \mathrm{C}$ (hyperthermia) has been shown to induce apoptosis in cancer. NIR agents have been extensively used for image-guided effective therapy. ${ }^{6-9}$ The fluorescence based NIR materials are in great demand as they are easily tracked using a fluorescence microscope. Photothermal agents like cyanine dyes have an inherent fluorescence property. Indocyanine green (ICG), an FDA approved fluorescent cyanine dye, has been extensively used as an imaging and photothermal agent due to its low cytotoxicity. However, poor

Department of Biosciences and Bioengineering, Indian Institute of Technology-Bombay, Powai, Mumbai-400076, India. E-mail: rsrivasta@iitb.ac.in; Tel: +912225767746

$\uparrow$ Electronic supplementary information (ESI) available: FTIR and UV-VIS data. See DOI: 10.1039/c5ra05997f solubility in water and low stability in the physiological system limit its use as an effective therapeutic agent. ${ }^{10}$ IR 820 has low cytotoxicity and a higher stability compared to ICG. ${ }^{\mathbf{1 1}}$ The fluorescent signal of IR 820 is much stronger than that of ICG in a mouse tail vein after $24 \mathrm{~h}$ of injection. Hence, IR 820 has been explored as a cost effective imaging agent. ${ }^{7,12-15}$ The heat generation property of IR 820 is nearly the same as ICG, making it suitable for image-guided photothermal therapy. The presence of a meso chlorine group in IR 820 facilitated the conjugation of chitosan to IR 820. Chitosan-conjugated IR 820 showed better cytotoxicity compared to the free dye in MES-SA and DX5 cancer cells. ${ }^{16}$ A similar study was done with PEG diamine conjugated to IR 820. Less PEG diamine-conjugated IR 820 accumulated in kidney and lung tissue than free IR 820 in mice, suggesting the importance of IR 820 nanoformulation in cancer therapeutics. ${ }^{7}$

Although PTT is an effective therapy, to eliminate the recurrence of the tumour, chemotherapeutic agents/drugs have also been encapsulated or conjugated with either IR 820 or a IR 820 containing formulation. ${ }^{4,5,17,18}$ Owing to their biodegradable and biocompatible nature, the use of polymeric 
nanoformulations has been broadly explored. Currently PLGA, an FDA approved polymer, is being extensively used for the encapsulation of drugs and dyes., ${ }^{\mathbf{4 1 8 - 2 0}}$ The amphiphilic nature of this polymer makes it an ideal choice for the encapsulation of hydrophobic as well as hydrophilic molecules. However, the high cost of PLGA demands a cheaper alternative like PCL for novel therapeutic agents. In addition, most drugs are hydrophobic in nature and can be easily loaded on PCL. ${ }^{19}$ Its cost effectiveness and long degradation time makes it an ideal option for photo chemotherapy. To improve the efficacy of this polymer, the addition of an immunoadjuvant, GC, has been proposed for the generation of a self-immune response in the cell against cancer. GC, a water-soluble chitosan derivative, is preferred for the encapsulation of drugs at physiological pH. GC has been widely used for the delivery of DNA, anti-cancer agents, and dyes. ${ }^{20-24}$ GC has also been shown to enhance the fluorescence of the dye to make it a better imaging agent. ${ }^{24}$ To achieve a photothermal effect, we further chose IR 820. IR 820 has been shown to interact with nanoformulations resulting in an intrinsic peak shift from 820 to $652 \mathrm{~nm}$ in the case of the chitosan-IR 820 conjugate. ${ }^{16}$ Several peaks were observed upon the interaction of IR 820 with a IRPDcov (IR 820 conjugated PEG-diamine) nanoformulation. A probable explanation is that these peak shifts can be attributed to the extended $\pi$ system. $^{7}$ The significance of these interactions has not been studied in detail. Therefore, the primary goal in the development of a PCLGC-IR nanoformulation is to explore the IR 820 interaction and the significance of its optical and surface properties for efficient PTT in detail.

In our present study, we have formulated multifunctional PCLGC-IR composite nanoparticles for bioimaging and therapy. To our knowledge, we explored the significance of the IR 820 interaction with a PCLGC-IR nanoformulation by DSC, $\mathrm{XRD}$ and dual laser (750 $\mathrm{nm}$ and $808 \mathrm{~nm}$ ) for the first time. In addition to that, stability and degradation studies have been carried out for the first time using LCMS. Further, the cytotoxicity of the PCLGC-IR composite nanoparticles was evaluated using NIH3T3 cells. Cellular uptake and PTT studies were performed on MDA-MB-231 cells. Hence, we explored the multifunctionality of the PCLGC-IR composite nanoparticles as bioimaging, PTT, and chemotherapeutic agents.

\section{Experimental}

\section{Materials}

Polycaprolactone (PCL), poly (vinyl alcohol) Mw: 13 000-23 000, 87-89\% hydrolyzed), glycol chitosan (GC) (MW: $100000-$ 300000 , degree of polymerization $\geq 400$, degree of purity $\geq 75$ $85 \%$ ), IR 820 dye, Hoechst 33342 and propidium iodide (PI) were purchased from Sigma-Aldrich. Acetone, dichloromethane, and methanol were purchased from Merck India Pvt. Ltd. DMEM, RPMI 1640, antibiotic-antimycotic solution, phosphate buffer saline (PBS) with calcium and magnesium salts and trypsin-EDTA solutions were purchased from Himedia Pvt Ltd Mumbai India. All the chemicals and reagents used were of analytical grade. MDA-MB-231 cells were purchased from NCCS Pune, India.

\section{Formulation of PCLGC-IR 820}

IR 820 loaded PCLGC-IR 820 nanoparticles were prepared using an oil in water $(\mathrm{o} / \mathrm{w})$ emulsion method. Briefly, $10 \mathrm{mg} \mathrm{mL}^{-1}$ PCL was dissolved in different ratio DCM-methanol mixtures along with $0.3 \mathrm{mg} \mathrm{mL}^{-1}$ IR 820 dye (ESI $\dagger$ ). To make the emulsion, the organic phase was slowly added into the aqueous phase containing $0.5 \%$ PVA and $0.05 \%$ GC in water (1:3 ratio). The emulsion was sonicated for $5 \mathrm{~min}$ followed by evaporation under magnetic stirring at room temperature. The NPs thus generated were centrifuged at $2500 \times g$ for $15 \mathrm{~min}$ to remove large particles and uncoated residues. Finally the NPs were collected by centrifugation at $12000 \times g$ for $20 \mathrm{~min}$ followed by three washes. To generate uniform separate nanoparticles, different ratios of the organic phase DCM-methanol and acetone-methanol and the aqueous phase were also tried.

\section{Characterization of PCLGC-IR 820}

The NP size distribution and poly dispersity were characterized by dynamic light scattering and their charge by using a zeta analyzer (Brookhaven Instruments Corporation, USA). The morphology and size were further analyzed using scanning electron microscopy (SEM, JSM 7600F Jeol) and transmission electron microscopy (TEM, Jeol 2100F). The absorbance spectra were obtained using a UV-visible spectrophotometer (Perkin Elmer lambda 25 USA). The samples were scanned from $400 \mathrm{~nm}$ to $1100 \mathrm{~nm}$ to determine the absorbance in the NIR range. The melting temperature of the NPs was determined using DSC. Two mg samples were subjected to scanning from $20^{\circ} \mathrm{C}$ to 100 ${ }^{\circ} \mathrm{C}$ with a heating rate of $20{ }^{\circ} \mathrm{C} \mathrm{min}^{-1}$. Fourier transform infrared spectroscopy (FTIR) was used to determine the chemical structure of the nanoparticles. The samples were loaded in KBr pellets. The spectra were recorded using a Magna 550 IR spectrophotometer (Nicolet Instrument Corporation). X-ray diffraction spectroscopy (XRD) was used to analyze the effect of IR 820 dye encapsulation on the composite nanoparticle molecular structure. The powder diffraction patterns were obtained using a Philips PW 3040/60 diffractometer with $40 \mathrm{kV}$, $30 \mathrm{~mA}$ graphite filtered $\mathrm{Cu} \mathrm{KR}$ radiation $(\lambda=0.1506)$. The data were obtained from scanning from $10^{\circ}$ to $80^{\circ}(2 \theta)$ at a scanning speed of 10 degrees per min.

\section{Determination of IR 820 encapsulation}

IR 820 encapsulation was analyzed in triplicate using UV-Vis spectroscopy. Briefly, a standard curve of IR 820 in a DCM and methanol mixture was plotted.

The lyophilized sample was dissolved in a DCM and methanol mixture. The sample was centrifuged at $15000 \mathrm{rpm}$ for 20 min to remove residual polymeric particles. The IR 820 concentration was determined from the standard curve plotted. The IR 820 entrapment and loading efficiency was calculated as follows:

$$
\text { Encapsulation efficiency }=\frac{\text { Amount of IR } 820 \text { in NPs }}{\text { Amount of IR } 820 \text { used in NPs }}
$$




\section{Stability and degradation studies}

The stability of the NPs over 10 days in the dark at $4{ }^{\circ} \mathrm{C}$ was determined using UV-visible spectrophotometry. The stability of the NPs was also checked in the presence of light and UV irradiation for $30 \mathrm{~min}$. The effects of various parameters such as light, UV irradiation and temperature, for $30 \mathrm{~min}$ and with a 808 $\mathrm{nm}(500 \mathrm{~mW})$ laser for $5 \mathrm{~min}$, on the degradation of the dye were determined. The degradation of the laser treated samples was determined by using both UV-visible spectroscopy and liquid chromatography-mass spectrophotometry (LCMS). The properties of the NPs following treatments were studied by LCMS to determine the precise changes. The NP stability was also evaluated using TEM and atomic force microscopy (AFM; Asylum research, USA) for any morphological changes.

\section{Photothermal effect}

The photothermal effect of the NPs was studied using a 750 and $808 \mathrm{~nm}$ infrared laser along with a digital thermocouple thermometer. Briefly $250 \mu \mathrm{L}\left(1000 \mu \mathrm{g} \mathrm{mL}{ }^{-1}\right)$ suspensions of the NPs were irradiated by either a 750 or $808 \mathrm{~nm}$ laser for $5 \mathrm{~min}$ in a 96 well plate inside a water bath maintained at $37^{\circ} \mathrm{C}$. The rise in temperature was measured at intervals of $1 \mathrm{~min}$.

\section{Cellular uptake and in vitro imaging}

MDA-MB-231 cells $\left(1 \times 10^{5}\right)$ were seeded in 12 well plates under $5 \% \mathrm{CO}_{2}$ at $37{ }^{\circ} \mathrm{C}$. After $24 \mathrm{~h}$, the cells were washed with PBS to remove unattached cells. The cells were incubated with media containing NPs for $4 \mathrm{~h}$. Rhodamine was adsorbed onto the NPs for visualization under a fluorescence microscope. The cells were washed thrice with phosphate buffered saline (PBS, $\mathrm{pH}$ 7.0) to remove any free NPs. Finally, PBS with $\mathrm{Ca}^{2+}$ and $\mathrm{Mg}^{2+}$ was added to the wells for live imaging under a Leica microscope (Germany) with $\lambda_{\mathrm{ex}} 528 \mathrm{~nm}$ and $\lambda_{\mathrm{ex}} 551 \mathrm{~nm}$. For quantitative analysis, cells were incubated with free IR 820 dye and PCLGCIR nanoparticles for $4 \mathrm{~h}$ in PBS with $\mathrm{Ca}^{2+}$ and $\mathrm{Mg}^{2+}$. At the end of the incubation period, the supernatant was collected and the absorbance was recorded at $820 \mathrm{~nm}$ in a Tecan multiplate reader. The percentage uptake was calculated using the formula:

$$
\begin{aligned}
& \% \text { uptake }= \\
& \frac{[\text { Total amount of dye used }]-[\text { Residual dye in supernatant }]}{\text { Total amount of dye used }} \times 100
\end{aligned}
$$

\section{Biocompatibility and photothermal studies on cells}

NIH3T3 $\left(1 \times 10^{4}\right)$ cells were seeded in 96 well plates and incubated for $24 \mathrm{~h}$ under $5 \% \mathrm{CO}_{2}$ at $37^{\circ} \mathrm{C}$. The medium was replaced with fresh complete media containing NPs $\left(100 \mu \mathrm{g} \mathrm{mL}^{-1}\right.$ to 1000 $\left.\mu \mathrm{g} \mathrm{mL}{ }^{-1}\right)$. The plate was further incubated for $24 \mathrm{~h}$. Further, the cells were analyzed for viability using an MTT assay.

For studying the photothermal effect, MDA-MB-231 $\left(1 \times 10^{4}\right)$ cells were seeded into a 96 well plate and incubated for $24 \mathrm{~h}$ under $5 \% \mathrm{CO}_{2}$ at $37{ }^{\circ} \mathrm{C}$. The medium was replaced with fresh media with $10 \%$ Fetal Bovine Serum (FBS) containing $125 \mu \mathrm{g}$ $\mathrm{mL}^{-1}$ of NPs and incubated for $4 \mathrm{~h}$. The cells were then washed with PBS and fresh complete media was added. Prior to laser application, the plate was placed in a dry bath incubator to maintain the temperature at $37{ }^{\circ} \mathrm{C}$. The cells were irradiated with a $808 \mathrm{~nm}$ laser $\left(4.2 \mathrm{~W} \mathrm{~cm}^{-2}\right)$ for $3 \mathrm{~min}$ on a $4 \mathrm{~mm}$ spot size. To determine the cytotoxic effect, the cells were further subjected to a MTT assay.

\section{Apoptosis study by Hoechst PI double staining}

For live/dead, apoptotic and necrotic cell visualization, MDAMB-231 $\left(1 \times 10^{5}\right)$ cells were seeded on a cover slip in a 12 well plate under $5 \% \mathrm{CO}_{2}$ at $37^{\circ} \mathrm{C}$. The remainder of the protocol was similar to that described earlier for the study of the photothermal effect. At the end of the laser application, the cells were stained with Hoechst $33342\left(10 \mu \mathrm{g} \mathrm{mL}^{-1}\right)$ and incubated at 37 ${ }^{\circ} \mathrm{C}$ for $20 \mathrm{~min}$ in PBS containing $\mathrm{Mg}^{2+}$ and $\mathrm{Ca}^{2+}$ ions. The cells were washed with PBS followed by propidium iodide $(10 \mu \mathrm{g}$ $\mathrm{mL}^{-1}$ ) staining for $5 \mathrm{~min}$ at $37^{\circ} \mathrm{C}$. The cells were washed thrice with PBS and visualized using a Leica fluorescence microscope (Germany).

\section{Results and discussion}

In this study, we have encapsulated IR 820 in PCLGC-IR composite nanoparticles. The GC coating on PCL made this composite amphiphilic for the successful encapsulation of both hydrophilic and hydrophobic drugs and small chemotherapeutic molecules such as siRNA. We prepared the composite nanoparticles using a single oil in water $(\mathrm{o} / \mathrm{w})$ emulsion method using $0.5 \%$ PVA as an emulsifier. Different ratios of DCM and methanol, and acetone and methanol were chosen to achieve uniform distribution. Polymers and dye dissolved in a $2: 1$ ratio of DCM to methanol and a 1:3 ratio of organic to aqueous phase resulted in uniform sphere shaped particles, as shown by the SEM analysis (ESI $\dagger$ ).

The size distribution of the NPs was around $150-200 \mathrm{~nm}$, as determined by the SEM analysis (Fig. 1a) while the hydrodynamic size was around $240 \mathrm{~nm}$ with a zeta charge of around +32 $\mathrm{mV} \pm 3 \mathrm{mV}$ (Table 1). Similar results were observed with ICG encapsulation in PLGA using 3\% PVA as an emulsifier. ${ }^{26}$ The small amount of PVA used in the preparation of the emulsion results in a high loading efficiency of the dye in the polymer. ${ }^{25}$ The encapsulation efficiency of dye was around $48 \%$. The PCLGC-IR particles were stable even at a low concentration of PVA. UV-visible absorbance studies (Fig. 1b) showed good stability of the dye inside the polymer for 10 days at $4{ }^{\circ} \mathrm{C}$ in the dark. The possible reason for the stability may be the IR 820 interaction with the nanoformulation. To explore the interaction of the IR 820 dye with the nanoformulation, UV-visible absorption, DSC and XRD measurements were taken. The UVvisible spectra of the PCLGC-IR composite nanoparticles showed two peaks: one at 845 and the other at 750 or $765 \mathrm{~nm}$ (Fig. 1b). A peak at $845 \mathrm{~nm}$ was common in all cases, whereas the presence of an additional peak varied depending on the aggregation of the NPs. A peak at $750 \mathrm{~nm}$ was observed for non- 
aggregated nanoparticles while aggregated nanoparticles showed a peak at around $760 \mathrm{~nm}$. The shift in peak from $820 \mathrm{~nm}$ to $845 \mathrm{~nm}$ and $750 / 760 \mathrm{~nm}$ may be attributed to the extended $\pi$ system. The peak shift has also been reported for a chitosan IR 820 conjugate. ${ }^{16}$

The interaction of IR 820 with the composite nanoformulation was analyzed by FTIR (ESI $\dagger$ ). The increase in the spectral intensity of the hydroxyl groups of PCL GC-IR at 2930 $\mathrm{cm}^{-1}$ and $3440 \mathrm{~cm}^{-1}$, the enhanced $\mathrm{C}=\mathrm{O}$ stretching at 1774 $\mathrm{cm}^{-1}$ and the enhanced peak at $1180 \mathrm{~cm}^{-1}$ were due to the addition of IR 820. The spectra compared to that of PCL GC suggested the encapsulation of IR 820 into PCL GC. A similar trend was observed for a chitosan IR conjugate. ${ }^{16}$ This infers that the IR 820 interaction with GC is much higher than the encapsulation in PCL.

Further, the stability and interaction of the dye with the nanoparticles have been studied using DSC. Blank NPs showed a melting temperature at around $55^{\circ} \mathrm{C}$ while the dye loaded NPs showed a melting temperature at around $56{ }^{\circ} \mathrm{C}$ (Fig. 2a). The DSC thermogram of the dye loaded NPs infers that dye encapsulation increases the stability of the particles. Similar thermal studies have been performed in a PCL gel polymer to study the effect of the interaction of metal with the PCL polymer gel. ${ }^{26}$

To validate the above results, XRD was performed to determine the phase change of the PCLGC-IR composite nanoparticles. PCL has two different characteristic peaks at Bragg's angles $2 \theta$ at $21.3^{\circ}$ and $23.6^{\circ}$ which suggest an orthorhombic unit cell like structure. ${ }^{27}$ The addition of GC at a small amount
Table 1 Hydrodynamic size and surface charge properties of the nanoparticles

\begin{tabular}{llll}
\hline Formulation & Size $(\mathrm{nm})$ & Polydispersity $(\mathrm{PDI})$ & Zeta potential(mV) \\
\hline PCL NP & $120 \pm 18$ & $0.194 \pm 0.005$ & $-20 \pm 4$ \\
PCLGC & $150 \pm 12$ & $0.221 \pm 0.04$ & $22 \pm 3$ \\
PCLGC-IR & $240 \pm 20$ & $0.158 \pm 0.034$ & $32 \pm 3$
\end{tabular}

did not affect the crystalline structure. However, IR 820 encapsulation in PCLGC-IR increases the intensity of the peak of Bragg's angle at $21.6^{\circ}$ (Fig. 2b). It indicates that the addition of either GC or IR 820 did not alter the crystal structure. These data suggest that IR 820 stabilizes the crystal structure of the PCLGC-IR composite nanoparticles. The interaction of IR 820 is a hydrophobic interaction with PCL while it is hydrophilic with GC. Hence, IR 820 holds the molecules tightly resulting in a more crystalline structure. IR 820 incorporation increases the surface charge of the PCLGC nanoparticles by $+10 \mathrm{mV}$. The MEP (Molecular Electrostatic Potential) analysis of the IR 820 dye showed a positive to negative charge polarity. ${ }^{28}$ So, it can interact with both positive and negatively charged molecules. To further validate the interaction of IR 820, spectroscopic analysis was performed for the different concentrations of PCL in the nanoformulation, keeping the dye and GC concentrations constant (ESI $\dagger$ ).

For photothermal transduction, the PCLGC-IR NPs, PCLGC NPs and water were irradiated with an $808 \mathrm{~nm}$ laser. The maximum temperature rise for the PCLGC-IR NPs was $46{ }^{\circ} \mathrm{C}$ at
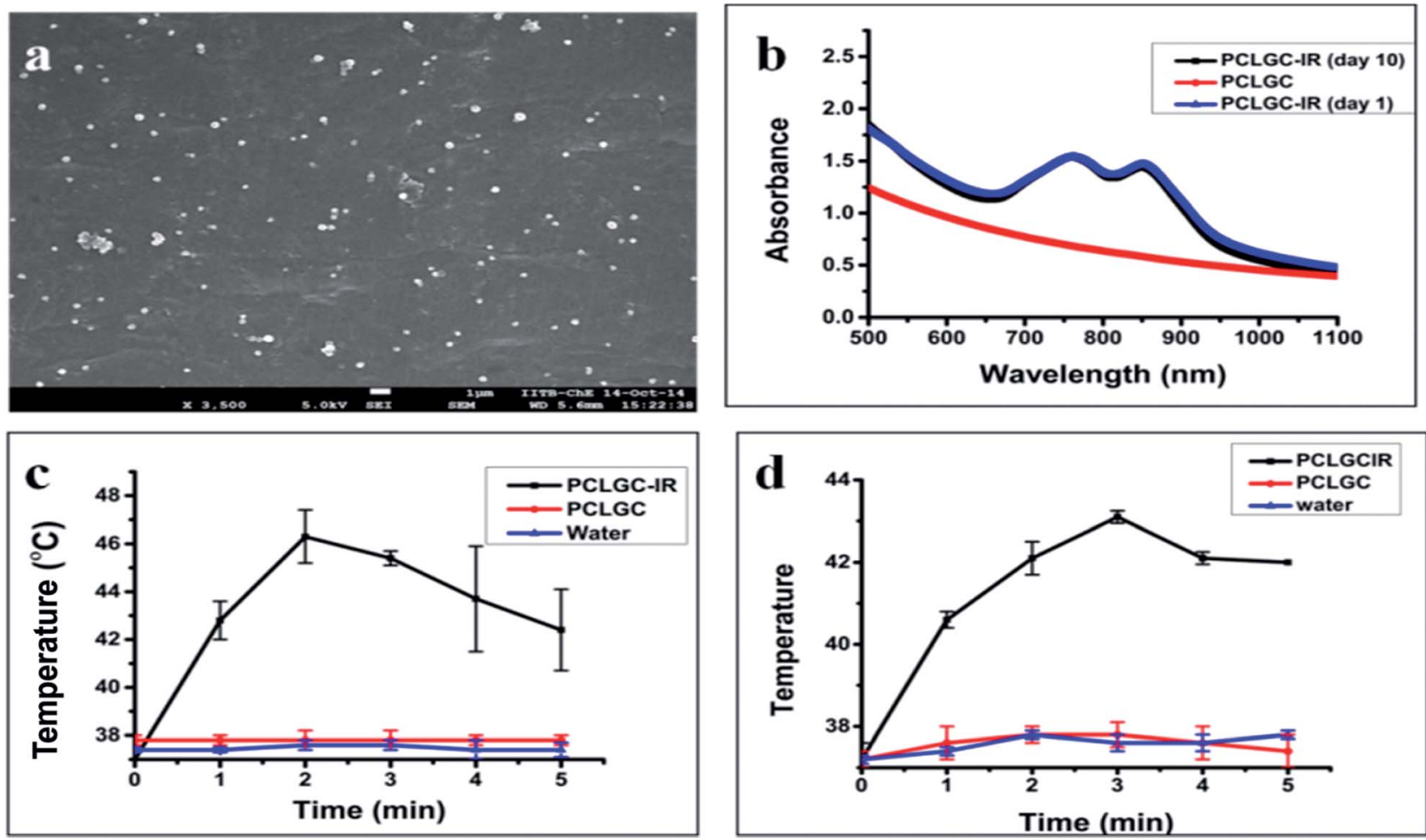

Fig. 1 (a) SEM image of the PCLGC-IR composite nanoparticles, (b) UV-visible absorbance of PCLGC and PCLGC-IR (day 1 and day 10), (c) the photothermal efficiency of PCLGC-IR, PCLGC and water under a $808 \mathrm{~nm}$ laser and (d) the photothermal efficiency of PCLGC-IR, PCLGC and water under a $750 \mathrm{~nm}$ laser $(n=3)$. 

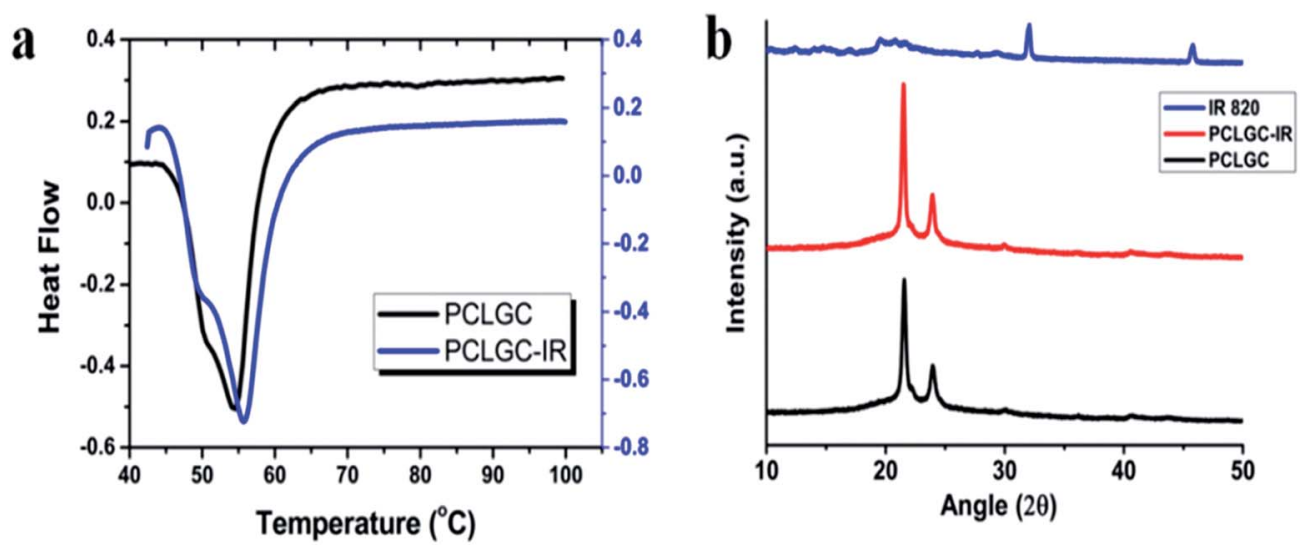

Fig. 2 (a) DSC curve of PCLGC and PCLGC-IR nanoparticles and (b) XRD data of PCLGC, PCLGC-IR and IR 820.

around $2 \mathrm{~min}$, which was followed by a drop in temperature suggesting degradation of the dye (Fig. 1c). The temperature remained above $42{ }^{\circ} \mathrm{C}$ until $5 \mathrm{~min}$. Supriya et al. have shown that $5 \mu \mathrm{M}$ of IR 820 dye conjugated to chitosan nanoparticles raised the temperature by $6{ }^{\circ} \mathrm{C}$ using a $8 \mathrm{~W} \mathrm{~cm}^{-2}$ laser, whilst in our case, we have used $4 \mathrm{~W} \mathrm{~cm}^{-2}$ laser to achieve the same temperature. ${ }^{15}$

This might be due to the stability of the dye inside the NPs as discussed above (Fig. 2b). Our results showed an additional absorbance peak at around $750-765 \mathrm{~nm}$. A similar shift was also observed with indocyanine dyes. However, there are no reports on the significance of these additional peaks. We also checked the photothermal effect of the PCLGC-IR NPs using a $750 \mathrm{~nm} 5$ $\mathrm{W} \mathrm{cm}{ }^{-2}$ laser as the UV-visible spectra showed an additional peak at $750 \mathrm{~nm}$. The maximum temperature rise was around 43 ${ }^{\circ} \mathrm{C}$ after $3 \mathrm{~min}$. The $750 \mathrm{~nm}$ laser treatment resulted in a constant temperature rise until $5 \mathrm{~min}$, making it suitable for PTT (Fig. 1d). Temperatures above $43{ }^{\circ} \mathrm{C}$ can lead to irreversible cytotoxic effects in cancer. In both cases, blank PCLGC NPs and water did not show any significant increase in temperature. This result infers the suitability of these NPs for dual laser treatment. In our case, the shift is in the infrared region $(750 \mathrm{~nm}$ and above) indicating the better efficacy of these NPs for photothermal therapy compared to agents described in previously reported articles. ${ }^{7,15}$

To analyze the effect of laser irradiation $(750 \mathrm{~nm}$ and 808 $\mathrm{nm}$ ), UV-visible and LCMS analyses were carried out. Interestingly, only one peak was diminished in the $750 \mathrm{~nm}$ treated sample, while in the $808 \mathrm{~nm}$ laser treated NPs, both peaks vanished suggesting the complete degradation of dye by the laser (Fig. 3a).

An $808 \mathrm{~nm}$ laser has the advantages of deeper penetration and safety. The fast rise in temperature caused by the $808 \mathrm{~nm}$ laser makes it suitable for use with a lower concentration of these PCLGC-IR composite nanoparticles. Hence, the rest of the experiments were performed using an $808 \mathrm{~nm}$ laser. Cliff yen et al. in 2008 used MS to detect the degraded product of IR 820 in dog plasma. A peak at around $330 \mathrm{Da}$ was reported in the case of degraded IR $820 .{ }^{29}$ However, the interaction of IR 820 in any nanoformulation has not been studied. Hence, extensive LCMS studies were performed to predict the effect of the laser on IR 820 degradation. To understand the mechanism of degradation, whether it's due to the high temperature or photo bleaching, the PCLGC-IR composite nanoparticles were subjected to TEM analysis to observe any morphological changes, and LCMS analysis for degradation studies. To rule out the possibility of temperature based degradation, the PCLGC-IR composite nanoparticles were also subjected to various conditions such as light and UV for $30 \mathrm{~min}$ and different temperatures for 5 min to predict the degradation mechanism (Table 2).

All the samples showed a major peak at around 573 Dalton (Da) except for the $808 \mathrm{~nm}$ laser treated samples. In the case of the $808 \mathrm{~nm}$ laser treated samples, a peak was observed at around $242 \mathrm{Da}$ (Table 2). It is difficult to analyze the dye when it is in the body once it degrades as it loses its fluorescence property. The peaks at $573 \mathrm{Da}$ and $242 \mathrm{Da}$ can be used to trace the dye inside the physiological system by mass spectrometry before and after laser irradiation (Fig. 4b). The peak at 573 Da may be due to a heterocyclic nitrogen ring that imparts fluorescence and an $808 \mathrm{~nm}$ absorbing compound. This also infers that the compound of molecular weight around $573 \mathrm{Da}$ is responsible for the photothermal effect. It indicates that the laser-based degradation of the dye is due to photo bleaching, as the different temperatures ranging from $37^{\circ} \mathrm{C}$ to $47^{\circ} \mathrm{C}$, applied for $5 \mathrm{~min}$, did not show any change.

However, longer incubation at higher temperatures degrades the dye. TEM analysis showed no morphological changes in the freshly prepared untreated and laser treated particles, suggesting these particles can also be used for controlled drug delivery (Fig. 4). A further study is going on to evaluate the significance of these degraded products and its significance for conjugation chemistry. We confirmed the effect of laser irradiation on the PCLGC-IR NPs after 10 days at room temperature under dark conditions using AFM (Fig. 5). AFM analysis revealed that the PCLGC-IR composite nanoparticles before laser irradiation are aggregated due to release of the IR 820 dye molecules (Fig. 5a), while no change was observed in the laser irradiated PCLGC-IR nanoparticles as the laser degraded the IR 820 dye (Fig. 5b). The PCLGC-IR composite nanoparticles remained morphologically intact after 12 days. The size distribution remained the same at 
a

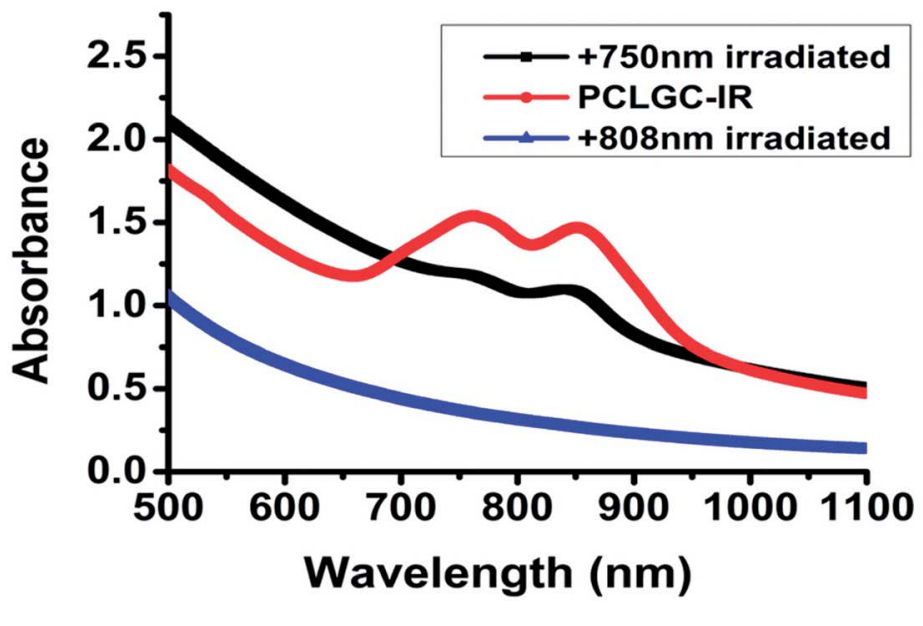

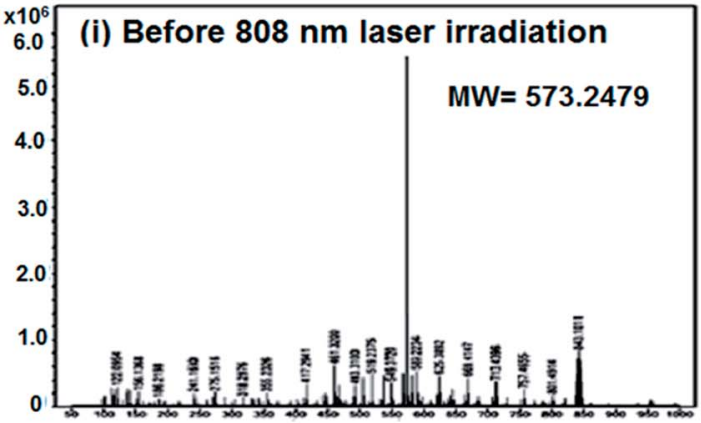

Molecular weight

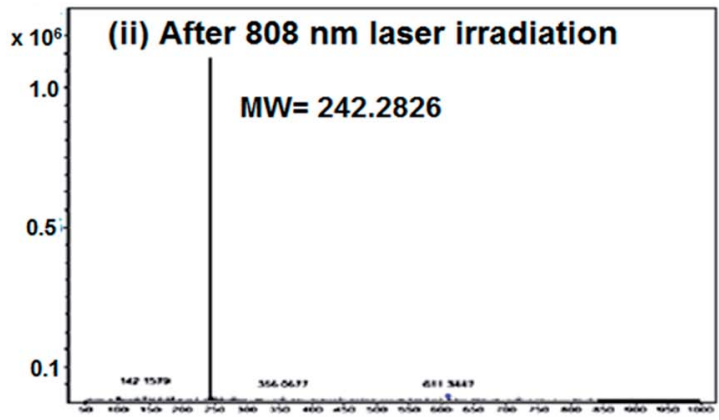

Molecular weight

Fig. 3 (a) The UV-visible absorbance of $808 \mathrm{~nm}$ and $750 \mathrm{~nm}$ laser treated PCLGC-IR composite nanoparticles. (b) (i) MS spectra of the PCLGCIR nanoparticles. (ii) MS spectra of the PCLGC-IR nanoparticles after $808 \mathrm{~nm}$ laser irradiation.

around $150-200 \mathrm{~nm}$. This further validates our hypothesis for the controlled release of therapeutics (drugs/nucleic acid) using the PCLGC-IR composite nanoparticles even after PTT.

IR 820 has a lower cytotoxicity than ICG and IR78 dyes. ${ }^{13}$ However, free IR 820 above $10 \mu \mathrm{mol}$ has been shown to be toxic to cells. ${ }^{15}$ The cytotoxicity of the PCLGC-IR composite nanoparticles was tested on NIH3T3 mouse fibroblast cells. Our results showed that the PCLGC-IR nanoparticles are non-toxic compared to free IR 820 (Fig. 6a). This suggests their suitability for in vivo studies. Further, the cellular localization of rhodamine labeled NPs was studied using a fluorescent
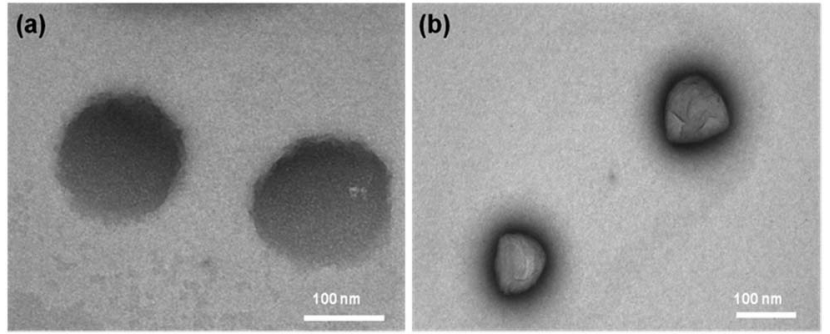

Fig. 4 (a) TEM image of PCLGC-IR before laser irradiation (b) TEM image of PCLGC-IR after $808 \mathrm{~nm}$ laser irradiation.

Table 2 MS spectra of the IR 820 dye in different conditions inside the composite nanoparticles

\begin{tabular}{llll}
\hline Sr. no. & Experimental conditions & MS spectra (Da) & Change in spectra (Da) \\
\hline 1 & $25^{\circ} \mathrm{C}$ for 30 min & 573 & Nil \\
2 & $37^{\circ} \mathrm{C}$ for 30 min & 573 & Nil \\
3 & Light for 30 min & 573 & Nil \\
4 & Near UV for 30 min & 573 & Nil \\
5 & UV for 30 min & 573 & Nil \\
6 & 808 nm laser irradiation for 5 min & 242 & 331 \\
7 & $40^{\circ} \mathrm{C}$ for 5 min & 573 & Nil \\
8 & $45^{\circ} \mathrm{C}$ & 573 & Nil \\
9 & $47^{\circ} \mathrm{C}$ & 573 & Nil
\end{tabular}


(a)
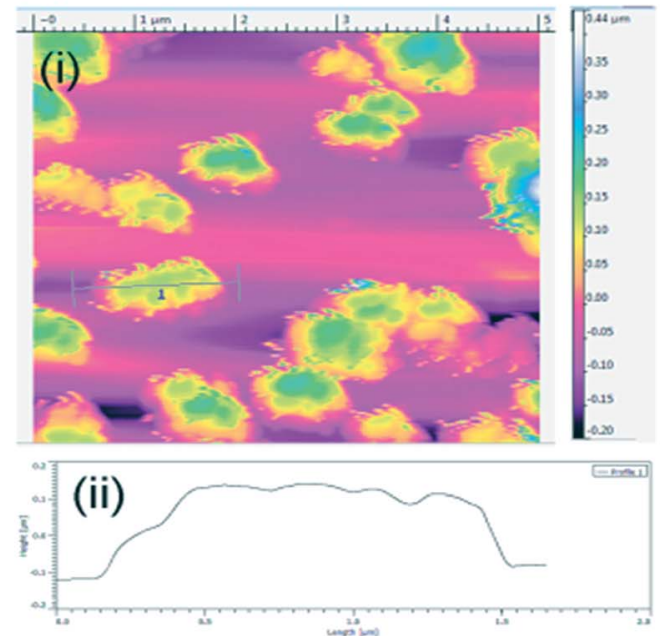

(iii)

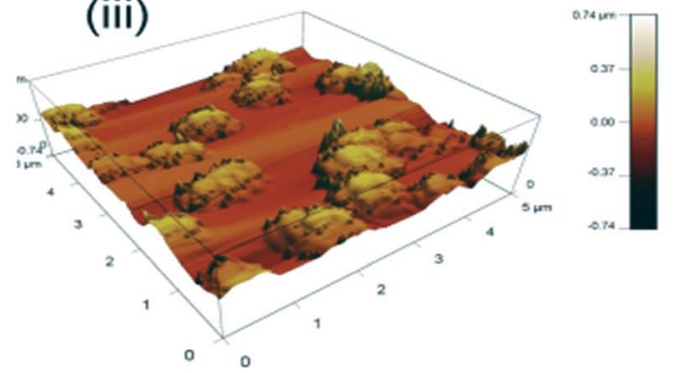

(b)
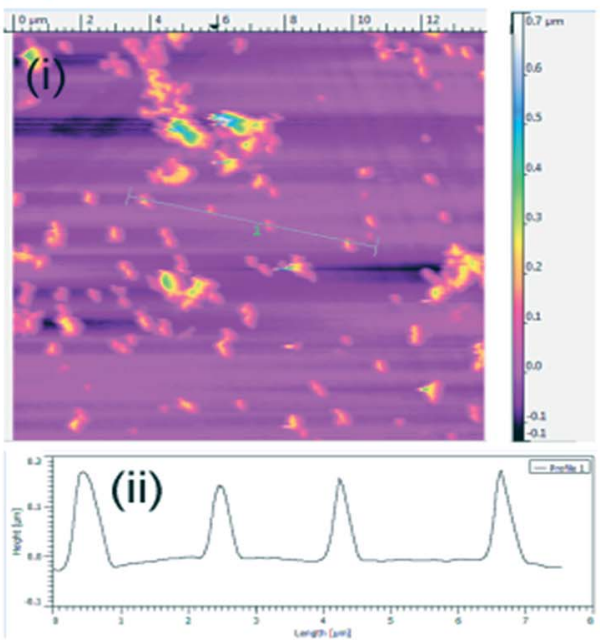

(iii)

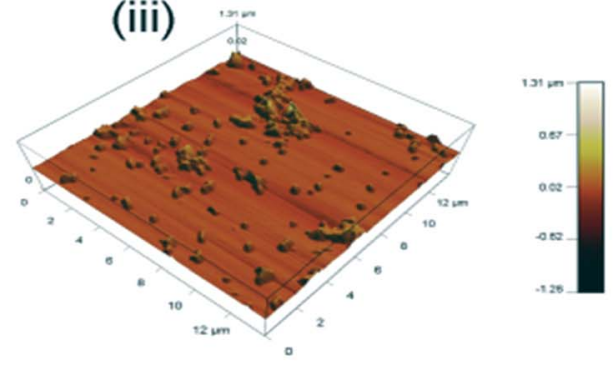

Fig. 5 AFM image of the PCLGC-IR composite nanoparticles before laser (ai) and post laser irradiation (bi) after 10 days. The height profile of the NPs ((aii) and (bii)) showing an average height of 150-200 nm. Corresponding 3D images ((aiii) and (biii)).
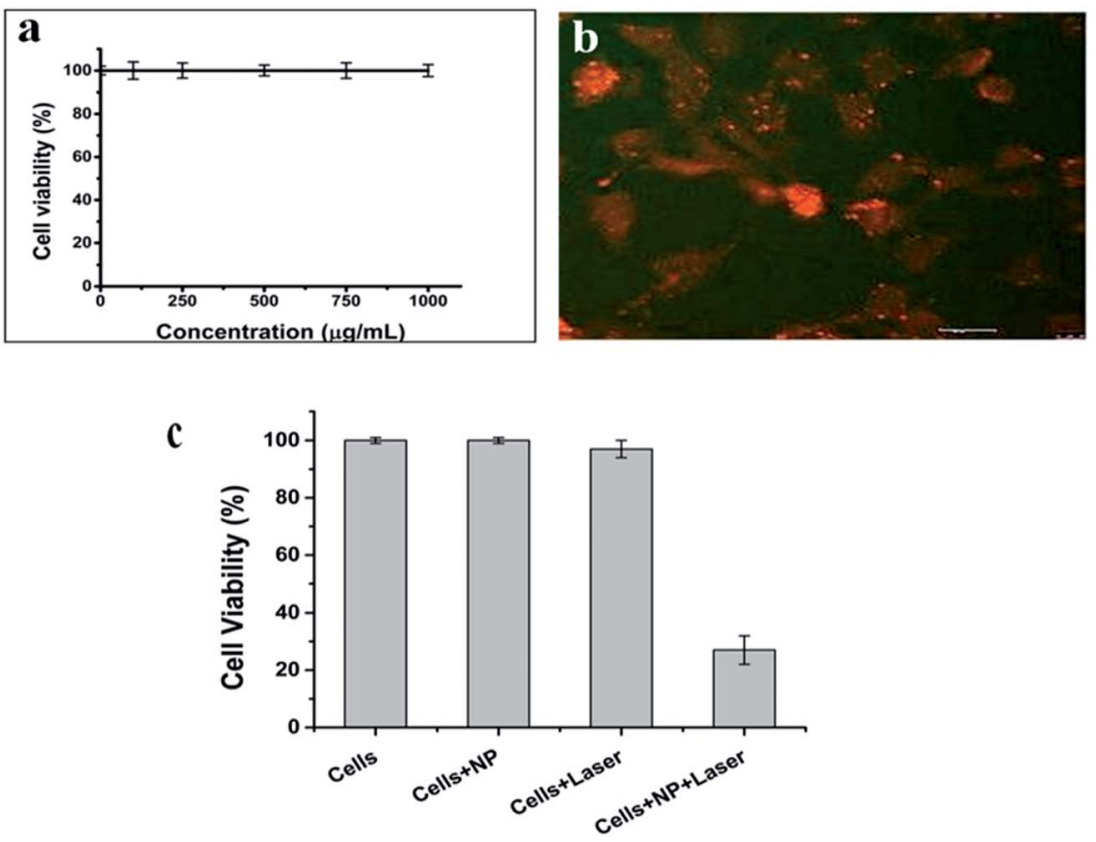

Fig. 6 (a) Biocompatibility of PCLGC-IR in NIH3T3 cells. (b) Cellular uptake of rhodamine labeled PCLGC-IR, and (c) photothermal therapy of PCLGC-IR with an $808 \mathrm{~nm}$ laser in MDA-MB-231 cells. 


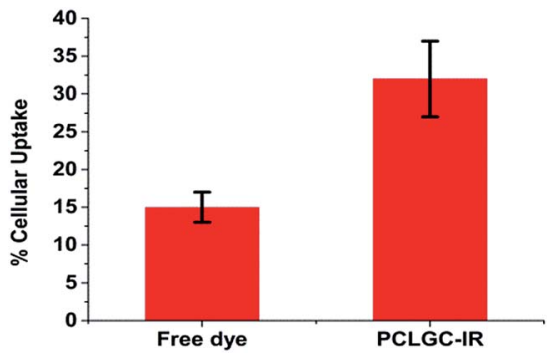

Fig. 7 Percentage cellular uptake of free dye and PCLGC-IR in MDAMB 231 cells.

microscope (Fig. 6b). The enhanced cellular uptake can be attributed to the interaction between the positively charged glycol chitosan coating and the negatively charged cancer cell surface. The cellular uptake of the PCLGC-IR nanoparticles was 2 times higher than that of free dye at the end of a $4 \mathrm{~h}$ time period (Fig. 7).

The in vitro photothermal effect was analyzed using MDAMB-231 cells (Fig. 6c). MDA-MB 231 is a triple negative breast cancer cell line. It is sensitive to hyperthermia. Since most of the triple negative breast cancer cells are getting drug resistant, we chose these cells lines to check the effectiveness of our NPs on them. Laser irradiated PCLGC-IR composite nanoparticles showed 3 fold reductions in cell viability in comparison to control cells. The significant hyperthermic cell death was observed due to a high cellular uptake of the positively charged PCLGC composite nanoparticles. To visualize the cell death, Hoechst 33342 and PI double staining were carried out (Fig. 8). The control samples did not show any PI stained cells inferring that the bare PCLGC NPs or the laser alone did not cause immediate cell death. However, cells treated PCLGC-IR NPs and a laser showed both apoptotic (deeply stained by Hoechst) and necrotic cells (stained with PI) indicating that the possible mechanisms of cell death are by both apoptosis and necrosis. The cytotoxicity of the PCLGC-IR composite nanoparticles can be further improved by combining chemotherapy. Thus, the PCLGC-IR composite nanoparticles can be effectively used for cancer theranostics.

\section{Cells $\quad$ Cells $+\mathrm{NP} \quad$ Cells + laser Cells $+\mathrm{NP}+$ laser}

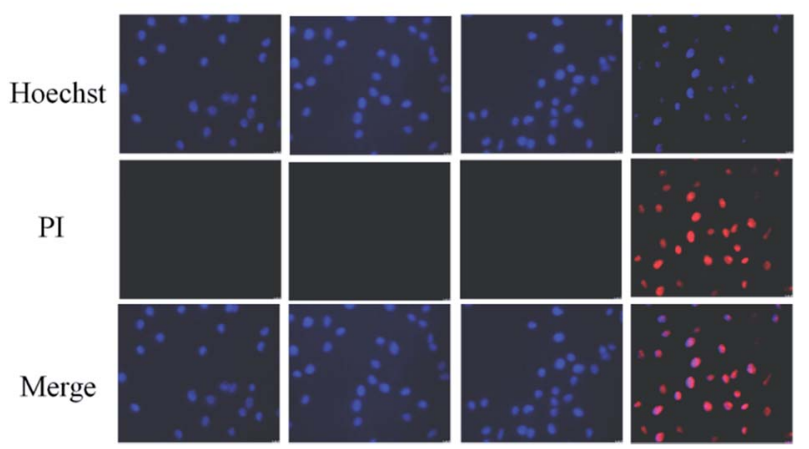

Fig. 8 Hoechst and PI staining of MDA-MB-231 cells in the presence and absence of $808 \mathrm{~nm}$ laser irradiation and PCLGC-IR.

\section{Conclusions}

We successfully formulated biocompatible and biodegradable PCLGC-IR composite nanoparticles for effective cancer theranostics. The IR 820 composite nanoparticles can be used for image-guided photothermal therapy. PCL and GC add the benefit of encapsulation of a chemotherapeutic molecule such as a drug along with a dye. IR 820 dye encapsulation enhanced the stability of the composite nanoparticles as observed by DSC. The stability of these NPs in aqueous solution makes them suitable for in vivo applications. Hence, we speculate that the PCLGC-IR composite nanoparticles can also be used for photothermal chemotherapy. GC also enhances the cellular uptake of these composite nanoparticles that leads to significant hyperthermic cell death in MDA-MB-231 cells. IR 820 has been shown to result in more than one peak upon encapsulation. In our case, two peaks were observed. For the first time, we studied how the photothermal effect affects the additional peak. These composite nanoparticles can be used with a dual laser system, $750 \mathrm{~nm}$ and $808 \mathrm{~nm}$ laser diode, for photo irradiation. We further studied the stability and degradation of the IR 820 dye in the composite nanoparticles by LCMS. The LCMS study can further help in analyzing the clearance of the dye through the physiological system. In summary, these composite nanoparticles can be used for multimodal cancer theranostics. Further detailed studies of the PCLGC-IR composite nanoparticles are underway to understand its true potential as a cancer theranostic agent.

\section{Note}

All experimental data have been represented as mean \pm S.D. $(P<$ $0.05)$ with $n=3$.

\section{Acknowledgements}

The authors would like to acknowledge SAIF-IITB and the chemical engineering department for characterization studies. We are thankful to Rohit Teotia, Rajan Singh and Narendra Jha for their help during the experiments. We are also thankful to Manish Kumar and M. Thakur for their help in writing manuscript. P. K. is thankful to the Indian Council of Medical Research, New Delhi for a senior research fellowship [(File no. 3/ 1/3/JRF-2008/HRD-102(32238)]. The authors also acknowledge IITB-Healthcare for financial support.

\section{References}

1 A. K. Rengan, A. B. Bukhari, A. Pradhan, R. Malhotra, R. Banerjee, R. Srivastava and A. De, Nano Lett., 2015, 15, 842-848.

2 W. Miao, G. Shim, S. Lee and Y. K. Oh, Biomaterials, 2014, 35, 4058-4065.

3 S. Z. Nergiz, N. Gandra, S. Tadepalli and S. Singamaneni, ACS Appl. Mater. Interfaces, 2014, 6, 16395-16402. 
4 M. Zheng, C. Yue, Y. Ma, P. Gong, P. Zhao, C. Zheng, Z. Sheng, P. Zhang, Z. Wang and L. Cai, ACS Nano, 2013, 7, 2056-2067.

5 P. Xue, K. K. Y. Cheong, Y. Wu and Y. Kang, Colloids Surf., B, 2015, 125, 277-283.

6 M. Zheng, P. Zhao, Z. Luo, P. Gong, C. Zheng, P. Zhang, C. Yue, D. Gao, Y. Ma and L. Cai, ACS Appl. Mater. Interfaces, 2014, 6, 6709-6716.

7 A. Fernandez, R. Manchanda and T. Lei, Int. J. Nanomed., 2014, 9, 4631-4648.

8 W. Guo, L. Zhang, J. Ji, W. Gao, J. Liu and M. Tong, Tumour Biol., 2013, 35, 3073-3078.

9 L. Wu, S. Fang, S. Shi, J. Deng, B. Liu and L. Cai, Biomacromolecules, 2013, 14, 3027-3033.

10 A. Fernandez-Fernandez, R. Manchanda, T. Lei, D. A. Carvajal, Y. Tang, S. Z. R. Kazmi and A. J. McGoron, Mol. Imaging, 2012, 11, 99-113.

11 D. S. Conceição, D. P. Ferreira and L. F. V. Ferreira, Int. J. Mol. Sci., 2013, 14, 18557-18571.

12 A. Manuscript, Changes, 2012, 29, 997-1003.

13 F. N. I. R. Fluorescent, J. L. Lakshmi, S. Bin Dolmanan and S. Tripathy, ACS Nano, 2013, 7, 6796-6805.

14 P. Huang, P. Rong, A. Jin, X. Yan, M. G. Zhang, J. Lin, H. Hu, Z. Wang, X. Yue, W. Li, G. Niu, W. Zeng, W. Wang, K. Zhou and X. Chen, Adv. Mater., 2014, 26, 6401-6408.

15 S. Srinivasan, R. Manchanda, A. Fernandez-Fernandez, T. Lei and A. J. McGoron, J. Photochem. Photobiol., B, 2013, 119, 5259.

16 Y. Yuan, Z. Wang, P. Cai, J. Liu, L.-D. Liao, M. Hong, X. Chen, N. Thakor and B. Liu, Nanoscale, 2015, 7, 3067-3076.
17 S. Su, Y. Tian, Y. Li, Y. Ding, T. Ji, M. Wu, Y. Wu and G. Nie, ACS Nano, 2015, 24, 1367-1378.

18 P. Zhao, M. Zheng, C. Yue, Z. Luo, P. Gong, G. Gao, Z. Sheng, C. Zheng and L. Cai, Biomaterials, 2014, 35, 6037-6046.

19 A. Kumari, S. K. Yadav and S. C. Yadav, Colloids Surf., B, 2010, 75, 1-18.

20 S. Srinivasan, R. Manchanda, T. Lei, A. Nagesetti, A. Fernandez-Fernandez and A. J. McGoron, J. Photochem. Photobiol., B, 2014, 136, 81-90.

21 H. Koo, K. H. Min, S. C. Lee, J. H. Park, K. Park, S. Y. Jeong, K. Choi, I. C. Kwon and K. Kim, J. Controlled Release, 2013, 172, 823-831.

22 S. Kim, D. J. Lee, D. S. Kwag, U. Y. Lee, Y. S. Youn and E. S. Lee, Carbohydr. Polym., 2014, 101, 692-698.

23 J. K. Rhee, O. K. Park, A. Lee, D. H. Yang and K. Park, Mar. Drugs, 2014, 12, 6038-6057.

24 S. Lee, S. W. Kang, J. H. Ryu, J. H. Na, D. E. Lee, S. J. Han, C. M. Kang, Y. S. Choe, K. C. Lee, J. F. Leary, K. Choi, K. H. Lee and K. Kim, Bioconjugate Chem., 2014, 25, 601-610. 25 A. P. Ranjan, K. Zeglam, A. Mukerjee, S. Thamake and J. K. Vishwanatha, Nanotechnology, 2011, 22, 295104.

26 C. P. Fonseca, F. Cavalcante Jr, F. A. Amaral, C. A. Z. Souza and S. Neves, Int. J. Electrochem. Sci., 2007, 2, 52-63.

27 M. Borjigin, C. Eskridge, R. Niamat, B. Strouse, P. Bialk and E. B. Kmiec, Int. J. Nanomed., 2013, 8, 855-864.

28 T. B. V. Neves and G. F. S. Andrade, J. Spectrosc., 2015, 805649.

29 C. Y. Chen, R. M. Fancher, Q. Ruan, P. Marathe, A. D. Rodrigues and Z. Yang, J. Pharm. Biomed. Anal., 2008, 47, 351-359. 